\title{
Spitzer Observations of M33 \& M83 and the Hot Star, H II Region Connection
}

\author{
R. Rubin ${ }^{1}$, J. Simpson ${ }^{1}$, S. Colgan ${ }^{1}$, R. Dufour ${ }^{2}$, R. Citron ${ }^{1}$, K. Ray ${ }^{1}$, \\ E. Erickson ${ }^{1}$, M. Haas ${ }^{1}$, and A. Pauldrach ${ }^{3}$ \\ ${ }^{1}$ NASA/Ames ${ }^{2}$ Rice University ${ }^{3}$ University Munich
}

\begin{abstract}
Emission-line observations made with the Spitzer Space Telescope are used to test the predicted ionizing spectral energy distributions of various stellar atmosphere models.
\end{abstract}

Keywords. ISM: abundances, H II regions, stars: atmospheres, galaxies: individual (M33, M83)

We observed emission lines of [S IV] 10.5, [Ne II] 12.8, [Ne III] 15.6, \& [S III $18.7 \mu \mathrm{m}$ from M33 and M83 cospatially with the Spitzer Space Telescope using the Infrared Spectrograph (IRS) in short-high mode (SH). Some results are shown in Figure 1.
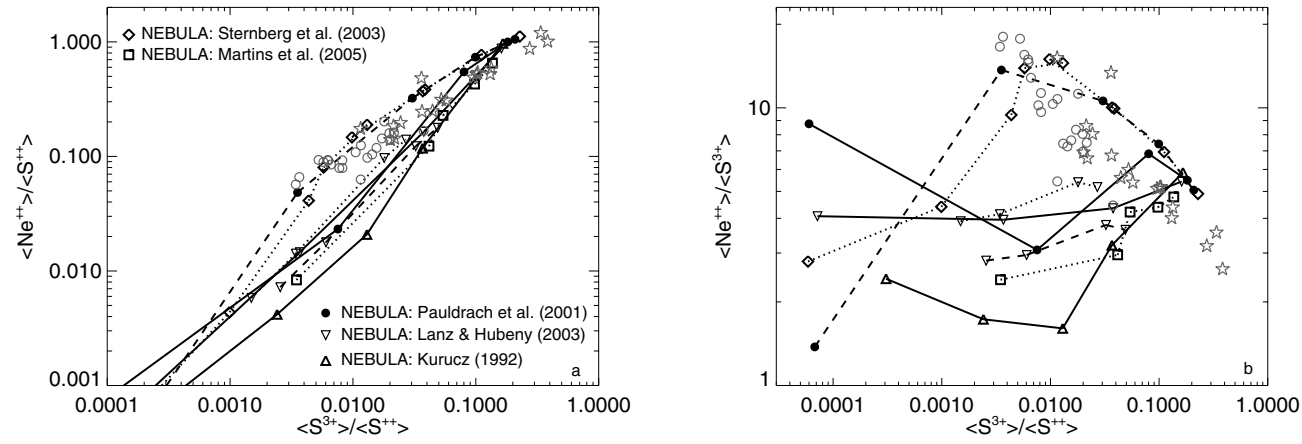

Figure 1. a) Theoretical predictions of the ratio of fractional ionizations $\left\langle\mathrm{Ne}^{++}\right\rangle /\left\langle\mathrm{S}^{++}\right\rangle$ vs. $<\mathrm{S}^{3+}>/<\mathrm{S}^{++}>$, computed using our photoionization code NEBULA (e.g., Rodríguez \& Rubin 2005). The lines connect the results of nebular models calculated using the ionizing SEDs predicted from various stellar atmosphere models as labeled, changing no other parameter except the SED. For the H II region models calculated with Pauldrach et al. (2001) atmospheres, the solid line connects models with dwarf atmospheres and the dashed line connects models with supergiant atmospheres. To compare our data with the models, we need to divide the observed $\mathrm{Ne}^{++} / \mathrm{S}^{++}$and $\mathrm{Ne}^{++} / \mathrm{S}^{3+}$ ratios by an assumed $\mathrm{Ne} / \mathrm{S}$ abundance ratio. We use the Orion Nebula $\mathrm{Ne} / \mathrm{S}=14.3$ (Simpson et al. 2004). The open circles (M83) and stars (M33) are derived from our observed line fluxes. b) The same as panel a) except the ordinate is $\left\langle\mathrm{Ne}^{++}\right\rangle /\left\langle\mathrm{S}^{3+}\right\rangle$. Both panels dramatically illustrate the sensitivity of the $\mathrm{H}$ II region model predictions of these ionic abundance ratios to the ionizing SED that is input to nebular plasma simulations. The M33 and M83 data lie closest to the Pauldrach et al. and Sternberg et al. (2003) supergiant loci.

\section{Acknowledgements}

Support is from Spitzer Space Telescope Cycle 1 and 2 programs 3412 and 20057.

\section{References}

Kurucz, R. L. 1992, in: B. Barbuy \& A. Renzini (eds.), IAU Symp. 149 p. 225

Lanz, T., \& Hubeny, I. 2003, ApJS 146, 417

Martins, F., Schaerer, D., \& Hillier, D. J. 2005 A $\mathscr{S} A$ 436, 1049

Pauldrach, A. W. A., Hoffmann, T. L., \& Lennon, M. 2001, A\&3A 375, 161

Rodríguez, M., \& Rubin, R. H. 2005, ApJ 626, 900

Simpson, J. P., Rubin, R. H., Colgan S. W. J., Erickson, E. F., \& Haas, M. R. 2004, ApJ 611, 338

Sternberg, A., Hoffmann, T. L., \& Pauldrach, A. W. A. 2003, ApJ 599, 1333 